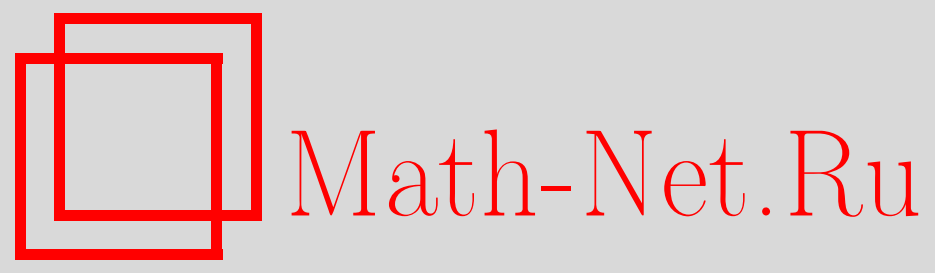

А. В. Нагаев, Большие уклонения для сумм решетчатых случайных величин при выполнении условия Крамера, Дискрет. матем., 1998, том 10, выпуск 3, 115-130

DOI: https://doi.org/10.4213/dm438

Использование Общероссийского математического портала Math-Net.Ru подразумевает, что вы прочитали и согласны с пользовательским соглашением http: //www . mathnet.ru/rus/agreement

Параметры загрузки:

IP : 3.85 .73 .92

26 апреля 2023 г., $17: 20: 59$ 


\title{
Большие уклонения пля сумм решетчатых случайных величин при выполнении условия Крамера
}

\author{
(C) 1998 г. $\quad$ A. B. Нагаев
}

\begin{abstract}
Рассматриваются суммы независимых одинаково распределенных решетчатых случайных величин. Предполагается, что одностороннее условие Крамера выполнено в некотором конечном интервале $[0, \lambda)$, то есть не существует крайнего правого сопряженного распределения. При дополнительных условиях регулярности, налагаемых на правый хвост исходного распределения, доказываются интегральная и локальная предельные теоремы о больших уклонениях любого порядка.
\end{abstract}

\section{1. Введение и основные результаты}

В настоящее время теория больших уклонений довольно развита и находит применение во многих разделах теории вероятностей. Классическая ее часть имеет дело с вероятностями больших уклонений сумм независимых случайных величин. В последующем она охватила гораздо более общие схемы. В наши планы не входит обзор этой теории. Желающие получить о ней более полное представление могут обратиться к [1-6].

Целью данной работы является решение одной задачи, связанной с классическим результатом Крамера [7].

Пусть случайные величины $\xi, \xi_{1}, \xi_{2}, \ldots$ независимы и имеют общее распределение $F(x)$. Положим

$$
\zeta_{n}=\xi_{1}+\ldots+\xi_{n}
$$

и

$$
F_{n}(x)=\mathbf{P}\left(\zeta_{n}<x\right), \quad P_{n}(x)=\mathbf{P}\left(\zeta_{n} \geqslant x\right) .
$$

Предположим, что выполнеко одностороннее условие Крамера, то есть

$$
0=\inf \left(s: \mathbf{E} e^{s \xi}<\infty\right)<\sup \left(s: \mathbf{E} e^{s \xi}<\infty\right)=\lambda<\infty .
$$

Условие (1) означает, что $P_{1}(x)$, правый хвост исходного распределения $F(x)$, показательно быстро убывает при $x \rightarrow \infty$. Через $f(s)$ обозначим производящую 
функцию $\xi$, то есть $f(s)=\mathrm{E} e^{s \xi}$. Согласно (1) $f(s)<\infty$, по меньшей мере, для $s \in(0, \lambda)$. Положим

$$
M(s)=f^{\prime}(s) / f(s), \quad M=\lim _{s \rightarrow \lambda} M(s)
$$

Поскольку

$$
M^{\prime}(s)=(f(s))^{-1} \int_{-\infty}^{\infty}(x-M(s))^{2} e^{s x} d F(x)>0
$$

уравнение $x=M(s)$ имеет единственный корень при $M(0)<x<M$. Следует заметить, что $M(0)$ или конечно, или равно $-\infty$.

Определим функцию уклонений

$$
\rho(x)=\inf _{0 \leqslant s<\lambda} f(s) e^{-s x}
$$

Легко видеть, что при $M(0)<x<M$

$$
\rho(x)=f(s(x)) \exp (-x s(x))
$$

Далее положим

$$
M_{\delta}= \begin{cases}M-\delta, & \text { если } M<\infty \\ 1 / \delta, & \text { если } M=\infty\end{cases}
$$

и

$$
m_{\delta}= \begin{cases}M(0)+\delta, & \text { если } M(0) \text { конечно } \\ -1 / \delta, & \text { если } M(0)=-\infty\end{cases}
$$

С этого момента $\delta$ служит символом произвольно малого числа. Так что вовсе необязательно, что в (4) и (5) $\delta$ одно и то же.

Следующая теорема хорошо известна (см., например, VIII. 4.2 в [8]).

Теорема 1. Пусть въполнено (1). Если $\xi$ принимает значения $a+k h, k \in \mathbf{Z}^{1}$, әде $h$ - махсималъный иаг распределения, то

$$
\mathbf{P}\left(\zeta_{N}=x\right)=B_{1}(x / n) n^{-1 / 2} \rho^{n}(x / n)(1+o(1)),
$$

moгда как

$$
P_{n}(x)=B_{2}(x / n) n^{-1 / 2} \rho^{n}(x / n)(1+o(1))
$$

равномерно по $x, x=n a+k h, m_{\delta} \leqslant x / n \leqslant M_{\delta}$, где

$$
B_{1}(x)=h\left(2 \pi M^{\prime}(s(x))\right)^{-1 / 2}, \quad B_{2}(x)=\left(2 \pi M^{\prime}(s(x))\right)^{-1 / 2} \frac{h}{1-e^{-s(x) h}},
$$

a $s(x)$ - корень уравнения $M(s)=x$ или, иначе, $s(x)=M^{-1}(x)$. 
В связи с теоремой возникает ряд вопросов. В частности, насколько можно расширить область уклонений, сохраняя (6) и (7); какая асимптотика возникает, когда $x / n<m_{\delta}$ или $x / n>M_{\delta}$, а (6) и (7) не выполняются; какая асимптотика ожидается при $\lambda=\infty$ ?

Случай $x / n<m_{\delta}$ изучался в [9] и [10]. В данной работе нас интересует случай $x / n>M_{\delta}$.

Легко предвидеть, что ширина области больших уклонений, допускающей крамеровскую асимптотику, зависит от регулярности изменения $P_{1}(x)$. Для абсолютно непрерывных распределений эта область оказывается неограниченной сверху при условии, что исходная плотность достаточно правильно ведет себя при $x \rightarrow \infty$. Некоторые условия регулярности такого рода были предложены в [11]. Что же касается решетчатых распределений, то ситуация тут совсем иная. Например, если

$$
\varliminf_{x \rightarrow \infty} \frac{-\ln P_{1}(x)}{x^{2}}>0
$$

то область уклонений в (6) и (7) не может быть расширена (см., например, [12]). Заметим, что три только что процитированные работы посвящены случаю $\lambda=\infty$.

Когда $\lambda<\infty$, приходится различать случаи $f(\lambda)<\infty$ и $f(\lambda)=\infty$. В данной работе наше внимание сосредоточено на втором из этих случаев.

Рассмотрим независимые одинаково распределенные случайные величины $\xi(s), \xi_{1}(s), \xi_{2}(s) \ldots$ с общей функцией распределения

$$
F_{s}(x)=\mathbf{P}(\xi(s)<x)=(f(s))^{-1} \int_{-\infty}^{x} e^{s u} d F(u), \quad 0<s<\lambda .
$$

Это распределение называется сопряженным к $F(x)$. Согласно (2)

$$
\mathbf{E} \xi(s)=M(s), \quad \mathbf{D} \xi(s)=M^{\prime}(s) .
$$

Если $f(\lambda)=\infty$, то не существует крайнего правого сопряженного распределения $F_{\lambda}(x)$. Однако при определенной правильности изменения $P_{1}(x)$ можно выбрать $a(s)$ и $b(s)$ таким образом, чтобы для $F_{s}(x b(s)+a(s))$ при $s \rightarrow \lambda$ существовало предельное распределение.

Определение 1. Скажем, что функция $h(x)$ принадлежит классу $\mathscr{S}$, если

$h(x)>0$ для $x \geqslant x_{0}>0$ и $h(\infty)=0$;

$h^{\prime \prime}(x) \downarrow 0$ и $x h(x) \rightarrow \infty$ при $x \rightarrow \infty$;

для $x \geqslant x_{0}$

$$
0 \leqslant-\frac{h^{\prime \prime}(x)}{h^{\prime}(x)} \leqslant \frac{2}{x}
$$

где $x_{0}$ - некоторая положительная постоянная.

Легко видеть, что этот класс довольно широк. Например, он содержит функции вида $x^{\alpha} m(x),-1 \leqslant \alpha<0$, где $m(x)$ медленно изменяется и является достаточно гладкой.

Обозначим

$$
p(x)=\mathbf{P}(\xi=x), \quad x \in \mathbf{Z}^{1}
$$


Определение 2. Скажем, что исходное распределение удовлетворяет условию $(S)$, если при $x \rightarrow \infty$

$$
p(x)=\exp \left(-\lambda x+\int_{x_{0}}^{x} h(u) d u\right)(1+o(1))
$$

где $h(x) \in \mathscr{S}$.

Определение 3. Скажем, что исходное распределение удовлетворяет условию $(R)$, если при $x \geqslant x_{0}$

$$
p(x)=x^{\alpha} m(x) e^{-\lambda x}
$$

где $\alpha>-1, m(x)$ медленно изменяется при $x \rightarrow \infty$ и $x_{0}-$ положительная постоянная.

Условия $(S)$ и $(R)$, в частности, означают, что

$$
\sup (s: f(s)<\infty)=\lambda, \quad \lim _{s \rightarrow \lambda} f(s)=\infty .
$$

При этом классы, определяемые этими условиями, по существу, включают в себя все распределения, имеющие правильно изменяющиеся хвосты, совместимые с (11).

Следующая теорема содержит основный результат данной работы.

Теорема 2. Если выполнено одно из условий $(S)$ или (R), то утверждение теоремъ 1 справедливо равномерно по $x, x=n a+k h, k \in \mathbf{Z}^{1}, x \geqslant n m_{\delta}$.

Стоит заметить, что аналогичное утверждение остается справедливым, когда исходное распределение абсолютно непрерывно с плотностью $p(x)$, удовлетворяющей либо $(S)$, либо $(R)$.

$\mathrm{B}$ дальнейшем $c$ означает любую положительную постоянную, конкретное значение которой не имеет значения. В частности, это означает, что $c+c=c, c^{2}=c$ и т. д. Через $\omega(t)$ мы обозначаем любую неотрицательную функцию со свойством $\omega(t) \rightarrow 0$, при $t \rightarrow \infty$, а через $\theta$ любую величину, изменяющуюся в $[-1,1]$.

\section{2. Основная лемма и доказательство теоремы 2}

Обозначим

$$
p_{s}(x)=e^{s x} p(x) / f(s), \quad 0 \leqslant s \leqslant \lambda
$$

и пусть $p_{n s}(x)$ является $n$-й сверткой $p_{s}(x)$. Следующая лемма играет главную роль в доказательстве теоремы 2 .

Основная лемма. Если въполнено одно из условий $(S)$ или $(R)$, то при $n \rightarrow \infty$

$$
\sup _{x}\left|\sqrt{n M^{\prime}(s)} p_{n s}(x)-\varphi\left((x-M(s)) / \sqrt{n M^{\prime}(s)}\right)\right|=o(1)
$$

равномерно по $s, 0<\delta \leqslant s \leqslant \lambda$. 
Здесь и далее $\varphi(u)$ обозначает плотность стандартного нормального распределения.

Доказательство этой леммы будет проведено позднее. Здесь мы покажем как из нее следует утверждение теоремы 2.

Пусть $\xi$ целочисленна. По определению

$$
\mathbf{P}\left(\zeta_{n}=x\right)=f^{n}(s) e^{-s x} p_{n s}(x),
$$

тогда как

$$
\mathbf{P}\left(\zeta_{n} \geqslant x\right)=f^{n}(s) \sum_{k \geqslant x} e^{-s k} p_{n s}(k)
$$

Положим $x=n M(s)$. Локальная часть теоремы немедленно следует из Основной леммы и (13). Чтобы доказать интегральную, запишем для произвольного $K>0$

$$
\begin{aligned}
\sum_{x \leqslant k \leqslant K} e^{-s x} p_{n s}(k) & =\frac{1}{\sqrt{n M^{\prime}(s)}} \sum_{x \leqslant k \leqslant K} e^{-s k} \varphi\left((k-n M(s)) / \sqrt{n M^{\prime}(s)}\right)+o\left(\frac{e^{-s x}}{\sqrt{n M^{\prime}(s)}}\right) \\
& =\frac{e^{-s x}}{\sqrt{2 \pi n M^{\prime}(s)}} \frac{1-e^{-s(K+1)}}{1-e^{-s}}+o\left(\frac{1}{\sqrt{n M^{\prime}(s)}}\right) .
\end{aligned}
$$

Очевидно,

$$
\sum_{k \geqslant x+K} e^{-s x} p_{n s}(k) \leqslant \frac{c}{\sqrt{n M^{\prime}(s)}} \sum_{k \geqslant x+K} e^{-s k}=\frac{c}{\sqrt{n M^{\prime}(s)}} e^{-s(x+K)} .
$$

Поскольку $K$ произвольно, получаем, что

$$
\sum_{k \geqslant x} e^{-s x} p_{n s}(k)=\frac{e^{-s x}}{\sqrt{2 \pi n M^{\prime}(s)}} \frac{1}{1-e^{-s}}(1+o(1)) .
$$

Теорема теперь следует из (14) и (15).

Если $\xi$ принимает значения $a+k h, 0<a<h, k \in \mathbf{Z}^{1}$, то утверждение Основной леммы приобретает вид

$$
\sup _{x}\left|\sqrt{n M^{\prime}(s)} p_{n s}(x)-h \varphi\left((x-M(s)) / \sqrt{n M^{\prime}(s)}\right)\right|=o(1),
$$

а в (15) вместо $1 /\left(1-e^{-s}\right)$ имеем $h /\left(1-e^{-s h}\right)$.

\section{3. Предварительные замечания по поводу доказательства Основной леммы}

Обозначим

$$
\begin{aligned}
& \varphi_{s}(t)=\mathbf{E} e^{i t \xi(s)}=\frac{f(s+i t)}{f(s)}, \\
& \bar{\varphi}_{s}(t)=\exp \left(-i t M(s) / \sqrt{M^{\prime}(s)}\right) \varphi_{s}\left(t / M^{\prime}(s)\right), \quad 0<s<\lambda .
\end{aligned}
$$


По формуле обращения

$$
p_{n s}(x)=\frac{1}{2 \pi} \int_{-\pi}^{\pi} e^{-i t x} \varphi_{s}^{n}(t) d t
$$

Предположим, что выполнены следующие условия : при $n \rightarrow \infty$

$$
\left(\bar{\varphi}_{s}\left(t n^{-1 / 2}\right)\right)^{n}=e^{-t^{2} / 2}+o(1)
$$

равномерно по $t,|t|<T$; для любого $0<\delta<\lambda$ существует $\eta=\eta(\delta)>0$ и $c=c(\delta)>0$ такие, что

$$
\sup _{\delta \leqslant s \leqslant \lambda}\left|\bar{\varphi}_{s}(t)\right| \leqslant e^{-t^{2} / 2}, \quad|t|<\eta
$$

и

$$
\sup _{\delta \leqslant s \leqslant \lambda} \sup _{\eta \leqslant|t| \leqslant \pi \sqrt{M^{\prime}(s)}}\left|\bar{\varphi}_{s}(t)\right|<1
$$

а для некоторого $n_{0} \geqslant 2$

$$
\varlimsup_{s \rightarrow \lambda} \int_{-\pi \sqrt{M^{\prime}(s)}}^{\pi \sqrt{M^{\prime}(s)}}\left|\bar{\varphi}_{s}(t)\right|^{n_{0}} d t<\infty
$$

При выполнении этих условий можно применить к (16) стандартную технику (см., например, локальную предельную теорему в [13]), непосредственно ведущую к утверждению Основной леммы.

\section{4. Доказательство Основной леммы при выполнении условия $(S)$}

Положим

$$
h(\tau, x)=\int_{x_{0}}^{x} h(u) d u-\tau x .
$$

Через $m(\tau)$ обозначим функцию обратную к $h(x)$. Пусть далее

$$
\sigma^{2}(\tau)=-m^{\prime}(\tau)
$$

Лемма 1. $E с л и ~ h(x) \in \mathscr{S}$, то для всех достаточно малых $\tau$ u $|u| \leqslant m(\tau) /(6 \sigma(\tau))$

$$
h(\tau, m(\tau)+u \sigma(\tau))=h(\tau, m(\tau))-u^{2} / 2+\varepsilon(\tau, u) u^{3}
$$

¿əe

$$
|\varepsilon(\tau, u)| \leqslant \varepsilon(\tau)=5 \sigma(\tau) /(6 m(\tau))
$$


Доказателъство. Рассмотрим функцию $-u^{2} h^{\prime}(u)$. Используя (10), получаем, что

$$
\left(-u^{2} h^{\prime}(u)\right)^{\prime}=-u^{2} h^{\prime}(u)\left(2 / u+h^{\prime \prime}(u) / h^{\prime}(u)\right) \geqslant 0
$$

то есть $-u^{2} h^{\prime}(u)$ не убывает. Поскольку $u h(u) \rightarrow \infty$ при $u \rightarrow \infty$, то $-u^{2} h^{\prime}(u) \rightarrow \infty$. Следовательно,

$$
\sigma(\tau) / m(\tau)=\left(m^{2}(\tau) h^{\prime}(m(\tau))\right)^{-1 / 2} \rightarrow 0 .
$$

Интегрируя в $(10)$ по $(x /(1+\delta), x)$, получаем, что

$$
0 \leqslant \ln \frac{h^{\prime}(x /(1+\delta))}{h^{\prime}(x)} \leqslant 2 \ln (1+\delta)
$$

или

$$
1 \leqslant \frac{h^{\prime}(x /(1+\delta))}{h^{\prime}(x)} \leqslant 1+3 \delta
$$

при условии, что $0<\delta \leqslant 1, x>2 x_{0}$. Поскольку $-h^{\prime}(u)$ не возрастает,

$$
-h^{\prime}(x /(1+\delta)) \geqslant-h^{\prime}(x(1-\delta / 2)) \text {. }
$$

Поэтому

$$
1 \leqslant \frac{h^{\prime}(x(1-\delta))}{h^{\prime}(x)} \leqslant 1+6 \delta
$$

при условии, что $0<\delta \leqslant 1 / 2, x>2 x_{0}$. Подобным образом для $0<\delta \leqslant 1 / 2$

$$
1-2 \delta \leqslant \frac{h^{\prime}(x(1+\delta))}{h^{\prime}(x)} \leqslant 1 .
$$

Итак,

$$
\left|h^{\prime}(x(1-\delta)) / h^{\prime}(x)-1\right| \leqslant 6 \delta,
$$

при условии, что $|\delta| \leqslant 1 / 6$. С учетом (10) имеем

$$
\left|h^{\prime \prime}(x+x \delta) / h^{\prime}(x)\right| \leqslant 5 / x, \quad|\delta| \leqslant 1 / 6 .
$$

В частности,

$$
\left|h^{\prime \prime}(m(\tau)+\theta u \sigma(\tau)) / h^{\prime}(m(\tau))\right| \leqslant 5 / m(\tau)
$$

при условии, что $|u| \leqslant m(\tau) /(6 \sigma(\tau))$.

Стоит напомнить, что $\sigma(\tau)=\left(h^{\prime}(m(\tau))\right)^{-1 / 2}$. Следовательно,

$$
h(\tau, m(\tau)+u \sigma(\tau))=h(\tau, m(\tau))-u^{2} / 2+\frac{h^{\prime \prime}(m(\tau)+\theta u \sigma(\tau))}{6\left(h^{\prime}(m(\tau))\right)^{3 / 2}} u^{3}
$$

Поскольку

$$
\frac{h^{\prime \prime}(m(\tau)+\theta u \sigma(\tau))}{6\left(h^{\prime}(m(\tau))\right)^{3 / 2}}=\theta(5 \sigma(\tau)) /(6 m(\tau))
$$

лемма доказана. 
Не теряя в общности, предположим, что $\xi$ и, следовательно, $\xi(s)$ целочисленны. Лемма 2. Положим $\tau=\lambda-s$ и пусть $\tau \rightarrow 0$. Тогда для любого фиксированного $\nu>0$

$$
\int|x-m(\tau)|^{\nu} e^{s x} d F(x)=\sqrt{2 \pi} b_{\nu}(\sigma(\tau))^{\nu+1} e^{h(\tau, m(\tau))}(1+o(1)),
$$

$u$ для любого челого фиксированного $\nu>0$

$$
\int(x-m(\tau))^{2 \nu+1} e^{s x} d F(x)=o\left((\sigma(\tau))^{2 \nu+2} e^{h(\tau, m(\tau))}\right),
$$

əдe

$$
b_{\nu}=\int|x|^{\nu} \varphi(x) d x, \quad \nu=0,1,2, \ldots
$$

Доказательство. Пусть $g_{\nu}(x)$ обозначает либо $|x|^{\nu}$, либо $x^{2 \nu+1}$. Выберем $X$ настолько большим, что для $x>X$

$$
p(x) \leqslant 2 \exp \left(-\lambda x+\int_{x_{0}}^{x} h(u) d u\right) .
$$

Запишем представление

$$
\int g_{\nu}((\approx-m) / \sigma) e^{s x} d F(x)=\Sigma_{1}+\Sigma_{2}+\Sigma_{3}+\Sigma_{4}
$$

где

$$
\begin{aligned}
& \Sigma_{1}=\int_{-\infty}^{X} g_{\nu}((x-m) / \sigma) e^{s x} d F(x), \\
& \Sigma_{2}=\int_{X}^{m-l \sigma} g_{\nu}((x-m) / \sigma) e^{s x} d F(x), \\
& \Sigma_{3}=\int_{m-l \sigma}^{m+l \sigma} g_{\nu}((x-m) / \sigma) e^{s x} d F(x), \\
& \Sigma_{4}=\int_{m+l \sigma}^{\infty} g_{\nu}((x-m) / \sigma) e^{s x} d F(x) .
\end{aligned}
$$

Здесь для краткости $m=m(\tau), \sigma=\sigma(\tau)$, а

$$
l=(\varepsilon(\tau))^{-\gamma}, \quad 0<\gamma<1 / 3,
$$

с тем же $\varepsilon(\tau)$, что и в лемме 1 . Последняя вместе с (21) приводят к соотношению

$$
\begin{aligned}
& e^{-h(\tau, m(\tau))} \Sigma_{3} \\
& =\sum_{|k-m| \leqslant l \sigma} g_{\nu}((k-m) / \sigma) \exp \left(-(1 / 2)((k-m) / \sigma)^{2}+\theta \varepsilon(\tau)((k-m) / \sigma)^{3}\right)(1+o(1)) .
\end{aligned}
$$

Принимая во внимание выбор $l$, продолжаем

$$
\Sigma_{3}=e^{h(\tau, m(\tau))} \sum_{|k-m| \leqslant l \sigma} g_{\nu}((k-m) / \sigma) \exp \left(-(1 / 2)((k-m) / \sigma)^{2}\right)(1+o(1)),
$$


откуда

$$
\Sigma_{3}=\sigma \sqrt{2 \pi} e^{h(\tau, m(\tau))}\left(\int_{-\infty}^{\infty} g_{\nu}(u) d u+o(1)\right) .
$$

Здесь существенно то, что $\sigma=\sigma(\tau) \rightarrow \infty$ при $t \rightarrow \infty$.

Суммы $\Sigma_{2}$ и $\Sigma_{4}$ оцениваются одинаково. Оценим, например, $\Sigma_{4}$. Поскольку $h(\cdot, x)$ выпукла, для $x \geqslant m+l \sigma$

$$
h(\tau, x) \leqslant h(\tau, m)+\frac{h(\tau, m+l \sigma)-h(\tau, m)}{l \sigma}(x-m) .
$$

Применение леммы 1 дает оценку

$$
h(\tau, x) \leqslant h(\tau, m)-\frac{l}{4 \sigma}(x-m)
$$

при условии, что $\tau$ достаточно мало.

Принимая во внимание (24), продолжаем

$$
\begin{aligned}
\Sigma_{4} & \leqslant 2 e^{h(\tau, m(\tau))} \sum_{k>m+l \sigma} g_{\nu}((k-m) / \sigma) \exp (-(l / 4)((k-m) / \sigma)) \\
& \leqslant c e^{h(\tau, m(\tau))} \sum_{k>m+l \sigma} \exp (-(l / 8)((k-m) / \sigma)) .
\end{aligned}
$$

Следовательно,

$$
\Sigma_{4}=o\left(\sigma e^{h(\tau, m(\tau))}\right) .
$$

Подобным же образом убеждаемся, что

$$
\Sigma_{2}=o\left(\sigma e^{h(\tau, m(\tau))}\right)
$$

Очевидно, что

$$
\Sigma_{1}=O\left(m^{\nu_{0}}\right)
$$

где

$$
\nu_{0}= \begin{cases}\nu, & \text { если } g_{\nu}(x)=|x|^{\nu}, \\ 2 \nu+1, & \text { если } g_{\nu}(x)=x^{2 \nu+1}\end{cases}
$$

С помощью правила Лопиталя находим, что

$$
\lim _{\tau \rightarrow 0} \frac{\ln m(\tau)}{h(\tau, m(\tau))}=-\lim _{\tau \rightarrow 0} \frac{m^{\prime}(\tau)}{m^{2}(\tau)}=0 .
$$

Это означает, что для любого $N$

$$
m^{N}=o\left(e^{h(\tau, m(\tau)}\right) .
$$

Следовательно,

$$
\Sigma_{1}=o\left(e^{h(\tau, m(\tau)}\right)
$$

Объединяя (25)-(29), приходим к желаемому результату. Лемма доказана. 
Полагая в $(22) \nu=0$, получаем, что

$$
f(s)=f(\lambda-\tau)=\sqrt{2 \pi} \sigma(\tau) e^{h(\tau, m(\tau))}(1+o(1) .
$$

Если же в (23) $\nu=0$, то ввиду (2), (10) и (30)

$$
\mathbf{E} \xi(s)=M(s)=m(\tau)+o(\sigma(\tau)) .
$$

Наконец, полагая в (22) $\nu=2,3$, находим, что

$$
\mathrm{D} \xi(s)=M^{\prime}(s)=\sigma^{2}(\tau)(1+o(1))
$$

и

$$
\varlimsup_{s \rightarrow \lambda} \mathbf{E}|\xi(s)-\mathbf{E} \xi(s)|^{3}(\mathbf{D} \xi(s))^{-3 / 2}<\infty
$$

Пусть

$$
\Delta_{s}(x)=\left|\sqrt{M^{\prime}(s)} p_{s}(x)-\varphi\left((x-M(s)) / \sqrt{M^{\prime}(s)}\right)\right| .
$$

Лемма 3. Если условие $(S)$ выполнено, то при $s \rightarrow \lambda$

$$
\sup _{x} \Delta_{s}(x)=o(1) \text {. }
$$

Доказательство. Будем писать для краткости $M, M_{1}, m$ и $\sigma$ вместо, соответственно, $M(s), \sqrt{M^{\prime}(s)}, m(\tau)$ и $\sigma(\tau)$. Если $|x-M| / M_{1}<X$, то ввиду (31) и (32) для всех достаточно малых $\tau$, по меньшей мере, выполнено неравенство $|x-m|<2 \sigma X$. Из (31), (32) и леммы 1 следует, что

$$
\begin{aligned}
h(\tau, x) & =h(\tau, m)-(1 / 2)((x-m) / \sigma)^{2}+o(1) \\
& =h(\tau, m)-(1 / 2)\left((x-M) / M_{1}\right)^{2}+o(1)
\end{aligned}
$$

равномерно по $x,|x-M| / M_{1}<X$. Из (12) и условия $(S)$ следует, что

$$
\sup _{|x-M| / M_{1}<X} \Delta_{s}(x)=o(1)
$$

Поскольку $h(\cdot, x)$ при $x>m$ убывает, то принимая во внимание (24), получаем оценку

$$
\sup _{x \geqslant M+X M_{1}} \Delta_{s}(x) \leqslant \varphi(X)+2\left(M_{1} / f(s)\right) e^{h\left(\tau, M+X M_{1}\right)}
$$

при условии, что $\tau$ достаточно мало. Используя (30), (32) и (34), находим, что

$$
\sup _{x \geqslant M+X M_{1}} \Delta_{s}(x) \leqslant 4 \varphi(X) .
$$

Подобным же образом находим, что

$$
\sup _{X \leqslant x \leqslant M-X M_{1}} \Delta_{s}(x) \leqslant 4 \varphi(X) .
$$

Очевидно, что

$$
\sup _{x \leqslant X} \Delta_{s}(x) \leqslant c M_{1} e^{\lambda X} / f(s)=\omega(1 / \tau) .
$$

Остается объединить полученные оценки. Лемма доказана. 
В частности, из леммы 3 следует, что при $s \rightarrow \lambda$

$$
\sup _{x}\left|F_{s}\left(u \sqrt{M^{\prime}(s)}+M(s)\right)-\Phi(u)\right|=o(1) .
$$

Из (33) немедленно следуют соотношения (17) и (18). Остается установить (19) и (20). Второе из этих соотношений прямо следует из леммы 3 , если заметить, что

$$
\frac{1}{2 \pi} \int_{-\pi}^{\pi}\left|\varphi_{s}(t)\right|^{2} d t=\sum_{x} p_{s}^{2}(x) \leqslant \sup _{x} p_{s}(x) .
$$

Для того чтобы установить (19), достаточно доказать, что

$$
\varlimsup_{s \rightarrow \lambda} \sup _{\eta \leqslant|t| \leqslant \pi \sqrt{M^{\prime}(s)}}\left|\varphi_{s}\left(t / \sqrt{M^{\prime}(s)}\right)\right|<1 .
$$

Из (35) следует, что для любого фиксированного $T>0$

$$
\varlimsup_{s \rightarrow \lambda} \sup _{\eta \leqslant|t| \leqslant T}\left|\varphi_{s}\left(t / \sqrt{M^{\prime}(s)}\right)\right|=e^{-\eta^{2} / 2} .
$$

Покажем, что

$$
\varlimsup_{s \rightarrow \lambda} \sup _{T \leqslant|t| \leqslant \pi \sqrt{M^{\prime}(s)}}\left|\varphi_{s}\left(t / \sqrt{M^{\prime}(s)}\right)\right|<1 .
$$

Очевидно, что

$$
1-\left|\varphi_{s}(t)\right|^{2}=2 \sum_{x} \sin ^{2}(t x / 2) \mathbf{P}\left(\xi(s)-\xi_{1}(s)=x\right) .
$$

Напомним, что $\xi(s)$ и $\xi_{1}(s)$ независимы и имеют общее распределение (8).

Пусть

$$
X_{t}=\left(x:|x|<\sqrt{M^{\prime}(s)}, \min _{k \in \mathbf{Z}^{1}}|t x-2 \pi k| \geqslant \pi / 4\right) .
$$

Тогда

$$
1-\left|\varphi_{s}(t)\right|^{2} \geqslant 2 \sin ^{2}(\pi / 8) \sum_{x \in X_{t}} \mathbf{P}\left(\xi(s)-\xi_{1}(s)=x\right) .
$$

Далее,

$$
\sum_{x \in X_{t}} \mathbf{P}\left(\xi(s)-\xi_{1}(s)=x\right) \geqslant\left|\left(x: x \in X_{t}\right)\right| \min _{|x| \leqslant \sqrt{M^{\prime}(s)}} \mathbf{P}\left(\xi(s)-\xi_{1}(s)=x\right) .
$$

Из леммы 3 следует, что

$$
\min _{|x| \leqslant \sqrt{M^{\prime}(s)}} \mathbf{P}\left(\xi(s)-\xi_{1}(s)=x\right)=\frac{1}{\sqrt{4 \pi}} e^{-1 / 4}+o(1) .
$$

При этом

$$
\varliminf_{s \rightarrow \lambda} \frac{\left|\left(x: x \in X_{t}\right)\right|}{\sqrt{M^{\prime}(s)}}>0
$$

равномерно по $t,|t| \geqslant T / \sqrt{M^{\prime}(s)}$. Тем самым, (19) установлено и, следовательно, Теорема 2 доказана. 


\section{5. Доказательство Основной леммы при выполнении условия $(R)$}

Пусть $l(x)$ обозначает любую функцию со свойством

$$
\lim _{x \rightarrow \infty} l(x) / m(x)=1 \text {. }
$$

Следующая лемма яаляется аналогом леммы 2.

Лемма 4. Если условие $(R)$ выполнено, то для любого фиксированного $\nu \geqslant 0$ при $s \rightarrow \lambda$

$$
\sum_{x} x^{\nu} e^{s x} p(x)=\Gamma(\nu+\alpha+1) \tau^{-(\nu+\alpha+1)} l(1 / \tau), \quad \tau=\lambda-s
$$

Доказательство. Запишем для $\varepsilon>0, X>0$

$$
\sum_{x} x^{\nu} e^{s x} p(x)=\Sigma_{5}+\Sigma_{6}+\Sigma_{7}+\Sigma_{8}
$$

где

$$
\begin{aligned}
& \Sigma_{5}=\sum_{x \leqslant X} x^{\nu} e^{s x} p(x), \\
& \Sigma_{6}=\sum_{X<x<\varepsilon / \tau} x^{\nu} e^{s x} p(x), \\
& \Sigma_{7}=\sum_{\varepsilon / \tau \leqslant 1 /(\varepsilon \tau)} x^{\nu} e^{s x} p(x), \\
& \Sigma_{8}=\sum_{x>1 /(\varepsilon \tau)} x^{\nu} e^{s x} p(x) .
\end{aligned}
$$

Рассмотрим хорошо известное представление Караматы (см., например, VIII.9 в [14])

$$
m(x)=a(x) \exp \left(\int_{1}^{x} u^{-1} \omega(u) d u\right)
$$

где $a(x) \rightarrow a \neq 0$ при $x \rightarrow \infty$. Из этого представления следует, что для любого $\delta>0$ найдется $X>0$ такое, что

$$
\frac{1}{2} \min \left((x / y)^{\delta},(y / x)^{\delta}\right) \leqslant m(x) / m(y) \leqslant 2 \max \left((x / y)^{\delta},(y / x)^{\delta}\right)
$$

при условии, что $\min (x, y) \geqslant X$. Не теряя в общности, можно считать $X$ целым.

Очевидно,

$$
\Sigma_{5} \leqslant X^{\nu} e^{\lambda X}
$$


Согласно условию $(R)$

$$
\begin{aligned}
\Sigma_{7} & =\sum_{\varepsilon \leqslant x \tau \leqslant 1 / \varepsilon} x^{\nu+\alpha} e^{-\tau x} m(x) \\
& =m(1 / \tau) \sum_{\varepsilon \leqslant x \tau \leqslant 1 / \varepsilon} x^{\nu+\alpha} e^{-\tau x}(1+o(1)) \\
& =\tau^{-(\nu+\alpha+1)} m(1 / \tau) \int_{\varepsilon}^{1 / \varepsilon} u^{\nu+\alpha} e^{-u} d u(1+o(1)) .
\end{aligned}
$$

В силу выбора $X$

$$
\Sigma_{6} \leqslant 2 m(1 / \tau) \tau^{-\delta} \sum_{X<x<\varepsilon / \tau} x^{\nu+\alpha-\delta} e^{-x \tau} .
$$

Если $-1<\nu+\alpha-\delta \leqslant 0$, то

$$
\sum_{X<x<\varepsilon / \tau} x^{\nu+\alpha-\delta} e^{-x \tau} \leqslant \int_{X-1}^{\varepsilon / \tau} u^{\nu+\alpha-\delta} e^{-\tau u} d u .
$$

Если $\nu+\alpha-\delta>0$, то

$$
\sum_{X<x<\varepsilon / \tau} x^{\nu+\alpha-\delta} e^{-x \tau} \leqslant \int_{X}^{\varepsilon / \tau+1} u^{\nu+\alpha-\delta} e^{-\tau u} d u
$$

при условии, что $\varepsilon$ достаточно мало. Так что

$$
\sum_{X<x<\varepsilon / \tau} x^{\nu+\alpha-\delta} e^{-x \tau} \leqslant \tau^{-(\nu+\alpha-\delta+1)} \omega(1 / \varepsilon) .
$$

Из (40) и (41) следует, что

$$
\Sigma_{6}=\theta m(1 / \tau) \tau^{-(\nu+\alpha+1)} \omega(1 / \varepsilon) .
$$

Далее,

$$
\Sigma_{8} \leqslant 2 m(1 / \tau) \tau^{\delta} \sum_{x \tau>1 / \varepsilon} x^{\nu+\alpha+\delta} e^{-x \tau} .
$$

Повторяя рассуждения, приводящие к (42), получаем, что

$$
\Sigma_{8}=\theta m(1 / \tau) \tau^{-(\nu+\alpha+1)} \omega(1 / \varepsilon) .
$$

Остается собрать вместе $(36),(38),(39),(42)$ и (43) и принять во внимание произвольность $\varepsilon$. Лемма доказана.

Из леммы 4 следует, что при $s \rightarrow \lambda$

$$
\begin{aligned}
f(s) & =\Gamma(\alpha+1) \tau^{-(\alpha+1)} l(1 / \tau), \\
\tau^{\nu} \mathbf{E} \xi^{\nu}(s) & =\frac{f^{(\nu)}(s)}{f(s)}=\frac{\Gamma(\nu+\alpha+1)}{\Gamma(\alpha+1)}+o(1), \\
\mathbf{D} \xi(s) & =(\alpha+1) \tau^{-2}(1+o(1)), \\
\mathbf{E}|\xi(s)|^{3} & =(\alpha+3)(\alpha+2)(\alpha+1) \tau^{-3}(1+o(1)) .
\end{aligned}
$$


Из (44) немедленно получаем (33) и, следовательно, (17) и (18).

Более того, из (44) следует, что все моменты $\tau \xi(s)$ при $\tau \rightarrow 0$ сходятся к соответствующим моментам стандартного гамма-распределения с параметром формы $\alpha+1$.

Пусть

$$
g_{\alpha}(x)= \begin{cases}x^{\alpha} e^{-x} / \Gamma(\alpha+1), & \text { если } x>0, \\ 0, & \text { если } x \leqslant 0 .\end{cases}
$$

Лемма 5. Если условие $(R)$ выполнено, то для любого фиксированного $\eta>0$ при $\tau \rightarrow 0$

$$
\sup _{x \tau \geqslant \eta}\left|\tau^{-1} p_{s}(x)-g_{\alpha}(x \tau)\right|=o(1)
$$

Доказательство. Обозначим

$$
\Delta_{s}(x)=\left|\tau^{-1} p_{s}(x)-g_{\alpha}(x \tau)\right| .
$$

Из леммы 4 и условия $(R)$ следует, что

$$
\begin{aligned}
\sup _{\eta \leqslant x \tau \leqslant 1 / \varepsilon} \Delta_{s}(x) & =\sup _{\eta \leqslant x \tau \leqslant 1 / \varepsilon}\left|\tau^{-1} e^{-\tau x} x^{\alpha} m(x) / f(s)-g_{\alpha}(x \tau)\right| \\
& =\sup _{\eta \leqslant x \leqslant 1 / \varepsilon} g_{\alpha}(x)\left|\Gamma(\alpha+1) \tau^{(\alpha+1)} m(x / \tau) / f(s)-1\right|=o(1) .
\end{aligned}
$$

Применение (37) и (44) приводит к оценке

$$
\begin{aligned}
\sup _{x \tau \geqslant 1 / \varepsilon} \Delta_{s}(x) & \leqslant g_{\alpha}(1 / \varepsilon)+(f(s))^{-1} \tau^{-1} \sup _{x \tau \geqslant 1 / \varepsilon} e^{-\tau x} x^{\alpha} m(x) \\
& \leqslant g_{\alpha}(1 / \varepsilon)+c \sup _{x \geqslant 1 / \varepsilon} e^{-x} x^{\alpha+\delta}=\omega(1 / \varepsilon) .
\end{aligned}
$$

Поскольку $\varepsilon$ произвольно, лемма доказана.

$\mathrm{C}$ помощью леммы 5 получаем, что

$$
\lim _{s \rightarrow \lambda} \sup _{\eta \leqslant|t| \leqslant T}\left|\varphi_{s}\left(t / \sqrt{M^{\prime}(s)}\right)\right|=|1-i \eta / \sqrt{\alpha+1}|^{-(\alpha+1)} .
$$

При выполнении условия $(R)(20)$ устанавливается так же, как это делалось при выполнении $(S)$. Так что остается доказать (19). Если $\alpha \geqslant 0$, то рассуждения, использованные в предыдущем параграфе не требуют изменений. Однако в случае, когда $-1<\alpha<0$, их следует слегка изменить.

Лемма 6. Пустъ в условии (R) $-1<\alpha<0 u 1<\gamma<-1 / \alpha$. Тогда

$$
\varlimsup_{s \rightarrow \lambda} \sum_{x}\left(p_{s}(x)\right)^{\gamma}=0 .
$$

Доказательство. Запишем

$$
\sum_{x}\left(p_{s}(x)\right)^{\gamma}=\Sigma_{9}+\Sigma_{10}+\Sigma_{11}
$$


где

$$
\begin{aligned}
\Sigma_{9} & =\sum_{x \leqslant X}\left(p_{s}(x)\right)^{\gamma}, \\
\Sigma_{10} & =\sum_{X<x<1 / \tau}\left(p_{s}(x)\right)^{\gamma}, \\
\Sigma_{11} & =\sum_{x>1 / \tau}\left(p_{s}(x)\right)^{\gamma}
\end{aligned}
$$

Первое из соотношений (44) приводит к оценке

$$
\Sigma_{9} \leqslant(f(s))^{-\gamma} e^{\lambda \gamma X} \sum_{x \leqslant X}\left(p_{s}(x)\right)^{\gamma} \leqslant c\left(\tau^{\alpha+1} / l(1 / \tau)\right)^{\gamma}
$$

Ввиду (37) и леммы 4

$$
\begin{aligned}
\Sigma_{10} & \leqslant 2(l(1 / \tau) / f(s))^{\gamma} \sum_{X<x<1 / \tau}\left(x^{\alpha}(x \tau)^{-\eta}\right)^{\gamma} \\
& \leqslant c \tau^{\gamma(\alpha+1-\eta)} \sum_{x \leqslant 1 / \tau} x^{\gamma(\alpha-\eta)} \leqslant c \tau^{\gamma-1}
\end{aligned}
$$

при условии, что $0<\eta<1 / \gamma+\alpha$. Наконец,

$$
\Sigma_{11} \leqslant c \tau^{\gamma-1} \sum_{x \tau>1}\left((x \tau)^{\alpha+\eta} e^{-\tau x}\right)^{\gamma} \tau \leqslant c \tau^{\gamma-1}
$$

Объединяя оценки и принимая во внимание выбор $\gamma$, получаем, что

$$
\sum_{x}\left(p_{s}(x)\right)^{\gamma} \leqslant c \tau^{\gamma-1}
$$

Лемма доказана.

Приступим к доказательству (19). Ясно, что только случай $-1<\alpha<0$ заслуживает нашего внимания. Пусть как и в лемме $61<\gamma<-1 / \alpha$. Удобно выбрать $\gamma$ так, чтобы $n_{0}=\gamma /(\gamma-1)$ было целым. Тогда из теоремы Хаусдорфа-Юнга (см., например, гл. XII в [15]) получаем оценку

$$
\frac{1}{2 \pi} \int_{-\pi}^{\pi}\left|\varphi_{s}(t)\right|^{n_{0}} d t \leqslant\left(\sum_{x}\left(p_{s}(x)\right)^{\gamma}\right)^{n_{0} / \gamma} .
$$

Теперь (19) непосредственно следует из леммы 6. Тем самым, теорема 2 доказана. 


\section{Список литературы}

1. Nagaev S. V. Large deviations of sums of independent variables. Ann. Prob.(1979) 7, 745789.

2. Розовский Л. В. Вероятности больших уклонений. Теория вероятн. и ее применения (1993) 38, 79-109.

3. Боровков А. А., Могульский А. А. Большие уклонения и проверка статистических гипотез. Труды Института математики СОРАН (1992) 2, 3-220.

4. Dembo A., Zeitouni O. Large Deviations Techniques and Applications. Jones and Bartlett, Boston, 1993.

5. Deuschel J. D., Strook D. W. Large Deviations. Academic Press, London, 1989.

6. Ellis R. S. Entropy, Large Deviations and Statistical Mechanics. Springer, Berlin, 1985.

7. Крамер Г. Об одной новой предельной теореме теории вероятностей. Успехи матем. паух (1944) 10, 166-178.

8. Петров В. В. Суммы независимых случайных величин. Наука, Москва, 1965.

9. Золотарев В. М. Об одной новой точке зрения на предельные теоремы, учитывающие большие уклонения. Труды 6-го Всесоюзного Совещания по теории вероятностей $и$ матем. статистике. Госиздат, Вильнюс, 1962, 43-47.

10. Ким Л. В., Нагаев А. В. О несимметричной проблеме больших уклонений. Теория вероятн. и ее применения (1975) 20, 58-68.

11. Нагаев А. В. Большие уклонения для одного класса распределений. В кн. : Пределъные теоремы теории вероятностей. Изд. АН УзССР, Ташкент, 1963, с.55-68.

12. Нагаев А. В., Локальные предельные теоремы с учетом больших уклонений. В кн.: Пределъные теоремъ и вероятностные прочессы. ФАН, Ташкент, 1967, с.71-88.

13. Гнеденко Б. В. Введение в теорию вероятностей. Наука, Москва, 1965.

14. Феллер В. Введение в теорию вероятностей и ее применения. Том 2. Мир, Москва, 1984.

15. Зигмунд А., Тригонометрические ряды. Том 2. Мир, Москва, 1965.

Статья поступила 18.03.1998. 